\title{
Dynamic and Rotatable Exchange Anisotropy in $\mathrm{Fe} / \mathrm{KNiF}_{3} / \mathrm{FeF}_{2}$ Trilayers
}

\author{
Stefania Widuch, Robert L. Stamps, Danuta Skrzypek and Zbigniew Celinski
}

Additional information is available at the end of the chapter

http://dx.doi.org/10.5772/56019

\section{Introduction}

Exchange bias with natural antiferromagnets is typically investigated for systems with only a single magnetic interface [1-7]. Of these, one of the best understood in terms of atomic level spin configurations is the epitaxial $\mathrm{Fe} / \mathrm{FeF}_{2}$ film system. The present work explores exchange bias in a structure in which an epitaxially grown $\mathrm{KNiF}_{3}$ film is used to separate the $\mathrm{Fe}$ and $\mathrm{FeF}_{2}$. The uniaxial magnetic anisotropy of the $\mathrm{KNiF}_{3}$ is much weaker than that of the $\mathrm{FeF}_{2}$, and the corresponding exchange bias and exchange anisotropies are also different.

By changing the thickness of the $\mathrm{KNiF}_{3}$, we show that it is possible to change the exchange anisotropy and bias from that of the $\mathrm{FeF}_{2}$ to that of the $\mathrm{KNiF}_{3}$. In the limit of very thick $\mathrm{KNiF}_{3}$, we observe unidirectional, uniaxial and rotatable exchange anisotropies. Competing effects from the $\mathrm{FeF}_{2}$ are observed as the $\mathrm{KNiF}_{3}$ thickness is reduced, allowing us to probe magnetic lengthscales associated with spin ordering near the $\mathrm{KNiF}_{3}$ interfaces.

We used ferromagnetic resonance (FMR) to obtain values for exchange anisotropies and bias. The FMR response is provided by the $\mathrm{Fe}$ in our $\mathrm{Fe} / \mathrm{KNiF}_{3} / \mathrm{FeF}_{2}$ systems, and magnetic anisotropies and other parameters are obtained by fitting the raw data to well known resonance conditions for thin films. In this chapter we show that the fitted values reveal an exchange anisotropy that appears to be dependent on applied field orientation relative to the magnetic anisotropy symmetry axes. Throughout the remainder of the chapter we label this anisotropy $H_{d y n}^{A F M}$. The onset of $H_{d y n}^{A F M}$ with temperature is similar to that of a rotatable anisotropy observed previously by us for $\mathrm{KNiF}_{3}$ systems $[8,9]$, and we suggest that $H_{d y n}^{A F M}$ in the present chapter consists of an isotropic 'rotatable' component $H_{\text {rot }}$ in addition to an orientation dependent, unidirectional component $H_{E}$.

It is useful at this point to summarize the relevant magnetic properties of the antiferromagnets and their associated exchange bias phenomena. $\mathrm{KNiF}_{3}$ is a Heisenberg 
antiferromagnet with cubic perovskite structure and Nèel temperature $\mathrm{T}_{\mathrm{N}}=250 \mathrm{~K}$ [10-13]. The $\mathrm{FeF}_{2}$ has a rutile-type crystal structure and Nèel temperature $\mathrm{T}_{\mathrm{N}}=79 \mathrm{~K}$ [14-17]. $\mathrm{KNiF}_{3}$ has a very small uniaxial anisotropy in comparison to $\mathrm{FeF}_{2}$ [12-17].

Results of magnetometry and FMR studies of bilayered thin films of single crystal $\mathrm{Fe}(001)$ and either single crystal or polycrystalline $\mathrm{KNiF}_{3}$ are given in references [8, 9]. The smallest lattice mismatch to the $\mathrm{Fe}$ is $1.2 \%$, assuming that the $\mathrm{Fe} / \mathrm{KNiF}_{3}$ interface coincides at the (100) face of both materials. This good lattice match between the ferromagnetic and antiferromagnetic layers preserves the cubic structure of both.

The (100) plane of $\mathrm{KNiF}_{3}$ should be compensated with both sublattices present in equal numbers. This should also be true for polycrystalline $\mathrm{KNiF}_{3}$ on Fe since all possible growth planes are reasonably well lattice matched with Fe. The most striking feature observed was a blocking temperature in the range $50 \mathrm{~K}$ to $80 \mathrm{~K}$. This temperature is much lower than the 250K Néel temperature expected for bulk $\mathrm{KNiF}_{3}$. Particularly relevant for the present work was the observation (by FMR) of a rotatable anisotropy that was an order of magnitude larger than the exchange bias.

As noted earlier, the $\mathrm{Fe} / \mathrm{FeF}_{2}$ system has been particularly well studied. Nogués et al. [1] have shown that the exchange bias depends strongly on the spin structure at the interface and in particular, on the angle between the FM and AFM spins. Work by Fitzsimmons et al. [18-22] using polarized neutron reflectometry and magnetometry show that spin configurations at the $\mathrm{FeF}_{2}$ interface differ from the bulk, and can be linked to exchange anisotropies and bias phenomena.

We have used MOKE microscopy to observe domains in a $\mathrm{Fe} / \mathrm{FeF}_{2}$ system to identify interface regions in which spin arrangements are distinct from either AFM or FM magnetic spin arrangements [23]. Results indicate that the crystallographic arrangement affects the value of the exchange bias but not the temperature dependence. Measurements of the temperature dependence of domain density in conjunction with coercivity field enhancement and exchange bias suggest that exchange bias and coercivity are different in origin in the sense that the unpinned magnetic moments at the AFM/FM interface are responsible for the enhancement of the coercivity field while pinned moments shifts the hysteresis loop.

In what follows we first discuss the sample structure and characterization in Section 2. This is followed in Section 3 by a discussion of reference FM/AFM1 and FM/AFM2 bilayers, and FM/AFM1/AFM2 trilayer results. In Section 4 we present our micromagnetic model and discuss the possibility of different thickness regimes. Results and discussion are summarized in Section 5.

\section{Sample preparation and structural characterization}

Samples were grown on GaAs(001) substrates using Molecular Beam Epitaxy. Wafers were annealed at elevated temperatures $\left(\sim 450^{\circ} \mathrm{C}\right)$ and sputtered with Ar ions until $4 \times 6$ reconstruction on the surface of GaAs was clearly visible. The Fe was deposited using K- 
cells and the fluorides were grown using e-beam evaporation at room temperature. A $0.6 \mathrm{~nm}$ thick Fe seed layer was followed by a $75 \mathrm{~nm}$ thick Ag buffer layer. At this point the structure was annealed at $350^{\circ} \mathrm{C}$ for $12 \mathrm{~h}$. From RHEED we confirmed that annealing produced an average terrace size of $\sim 13 \mathrm{~nm}$. Next an $\mathrm{Fe}(001)$ layer was grown at room temperature followed by a $\mathrm{KNiF}_{3}$ layer and topped with a $50 \mathrm{~nm} \mathrm{FeF}_{2}$ layer. Series with different $\mathrm{KNiF}_{3}$ thicknesses between 0 and $90 \mathrm{~nm}$ were prepared. The structures were capped with a $2.5 \mathrm{~nm}$ thick Au layer to protect samples during measurements in ambient conditions.

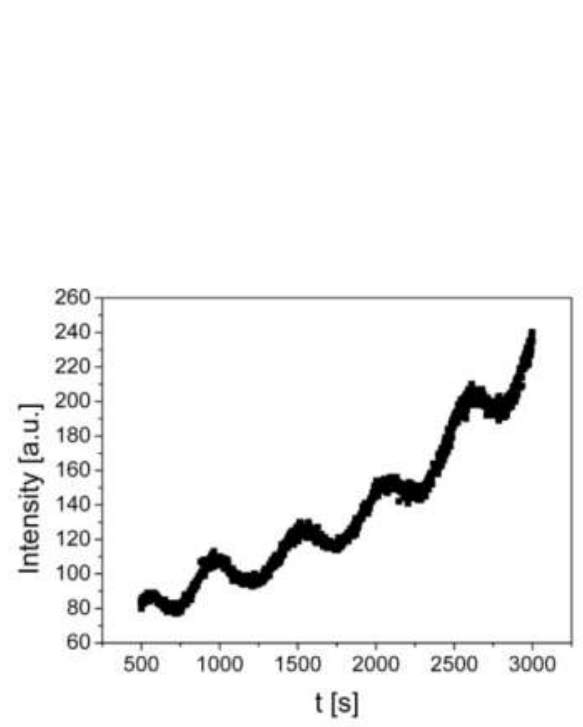

(a)

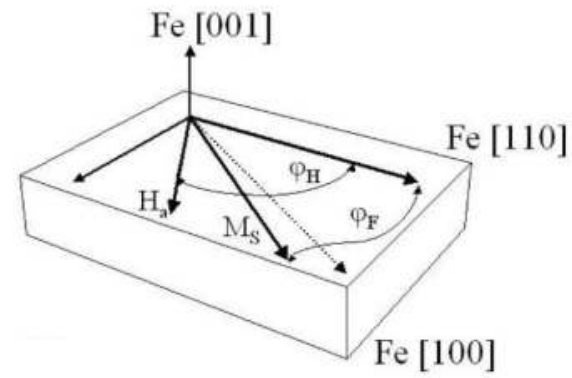

(c)

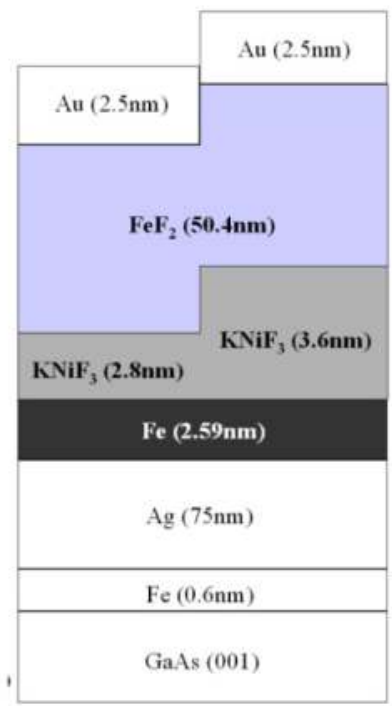

(b)

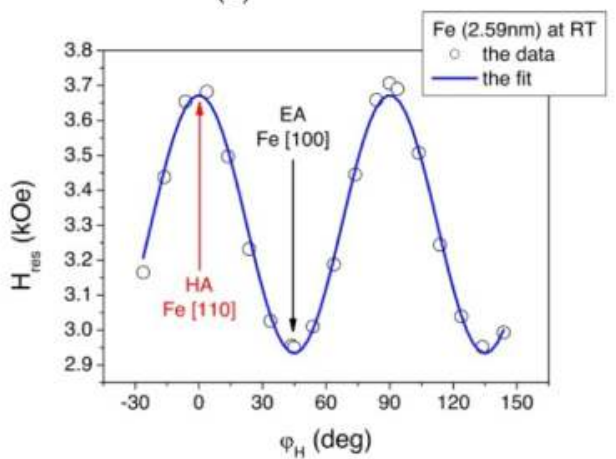

(d)

Figure 1. (a) Fragment of the RHEED intensity oscillations measured at the Fe specular spot during the growth; (b) a schematic diagram of the two grown $\mathrm{Fe} / \mathrm{KNiF}_{3} / \mathrm{FeF}_{2}$ structures with $2.8 \mathrm{~nm}$ - and $3.6 \mathrm{~nm}$ thick $\mathrm{KNiF}_{3}$; (c) geometry for FMR measurement; (d) resonance field for different orientations of the static applied field relative to a magnetocrystalline anisotropy axis for Fe thin film (2.59nm). Unfilled circles denote the data taken at RT. Solid line exhibits the fit. Fe easy axis [100] (denoted as EA) for $\varphi \mathrm{H}=$ $45^{\circ}$ and Fe hard axis [110] (denoted as HA) are shown. 
In figure 1(a), example RHEED intensity oscillations measured at the specular spot during the growth are shown, confirming layer by layer growth. Also, from RHEED we determined that the Fe layer is monocrystalline, and the fluorides layers are polycrystalline. Note that in order to reduce variations in growth conditions within the series, two thicknesses of $\mathrm{KNiF}_{3}$ were grown on each Fe film, thereby forming two samples. The geometry is sketched in figure $1(\mathrm{~b})$ for the 2.8 and $3.6 \mathrm{~nm}$ samples from the series. For each pair of samples the same roughness at the $\mathrm{Fe} / \mathrm{KNiF}_{3}$ interface is expected.

\section{Experimental details and results}

Ferromagnetic resonance was made at $24 \mathrm{GHz}$ at temperatures between $24 \mathrm{~K}$ and $300 \mathrm{~K}$, using the TE011 mode of a cylindrical cavity. The temperature dependence measurements were carried out in a dewar equipped with a closed-cycle helium refrigerator. The temperature of the sample cavity was monitored with two E-type thermocouples. The dc signal was measured on a diode, and the first derivative of the power absorption signal with respect to the applied field was detected. A lock-in amplifier technique was used employing a weak, $0.5 \mathrm{Oe}, 155 \mathrm{~Hz}$ ac modulation field superimposed on the applied dc magnetic field. The FMR absorption spectra were fit with standard Lorentzian function, providing directly the resonance field, $H_{r e s,}$ and FMR linewidth, $\Delta H$.

A sketch of the experimental geometry is shown in figure 1(c). In-plane angles for magnetization and applied field ( $\varphi_{F}$ and $\varphi_{H}$, respectively) are defined relative to the Fe[110] direction. Measurements were made by varying the angle $\varphi н$. An applied magnetic field was swept between $1-6 \mathrm{kOe}$. These fields were large enough to saturate the samples to within $2^{0}$ for all angles of applied field orientation. The samples were first measured at a room temperature, then were field cooled to $24 \mathrm{~K}$ in the cooling field $H_{c f}=0.87 \mathrm{kOe}$ and $H_{c f}=$ $2.07 \mathrm{kOe}$ for Fe easy axis (denoted as EA; $\varphi_{H}=45^{\circ}$ ) and Fe hard axis (denoted as HA; $\varphi_{H}=0^{0}$ ), respectively. These values of the cooling fields are significantly larger than field needed to saturate FM ( 600 Oe along hard axis).

The effective magnetization $4 \pi M_{e f f}$, and a fourfold anisotropy field, $H_{||}=\frac{2 K_{||}}{M_{S}}$ can be determined with FMR by measuring $H_{r e s}$ for different orientations of the static applied field $H_{\text {app }}$ relative to a magnetocrystalline anisotropy axis. An example for Fe thin film $(2.59 \mathrm{~nm})$ is shown in figure $1(\mathrm{~d})$. All FMR measurements were fit to the following resonance condition equation $[8,9]$ :

$$
\begin{aligned}
H_{r e s}= & \frac{1}{2}\left\{\left[\left(4 \pi M_{e f f}+\frac{3 H_{\mid \perp}}{4}\left(1+\cos 4 \phi_{H}\right)\right)^{2}+\left(\frac{2 \omega}{\gamma}\right)^{2}\right]^{1 / 2}-4 \pi M_{e f f}\right\} \\
& -H_{r o t}+\frac{H_{\mid I}}{8}\left(5 \cos 4 \phi_{H}-3\right)-H_{E} \cos \left(\phi_{H}-\phi_{E}\right)
\end{aligned}
$$


where the first term represents a constant shift in the resonance field baseline. This shift is due to the fact that $4 \pi M_{\text {eff }}$ and $\frac{\omega}{\gamma}$ are typically an order of magnitude larger than Fe fourfold anisotropy. The second term consists of an induced shift in the position of the resonance line due to exchange coupling which has been attributed to rotatable anisotropy. The third and fourth terms contain the angular variation of $H_{r e s}$ caused by the Fe fourfold and coupling induced unidirectional anisotropies, respectively. The easy direction of the unidirectional anisotropy is given by $\varphi_{E}$. Both terms $H_{r o t}$ and $H_{E}$ are temperature dependent. As noted above, Eq. (1) is valid only for $H_{r e s}$ large enough to saturate the magnetization.

A summary of results for effective magnetization and fourfold anisotropy fields determined from fits of $H_{\text {res }}$ to Eq. (1) are summarized in table 1.

The fourfold anisotropy field of the $18 \mathrm{ML}$ Fe films increased from 440 Oe (average value for all measured samples) at room temperature to $\sim 680$ Oe at $24 \mathrm{~K}$. For $36 \mathrm{ML}$ thick Fe layers, a similar increase of the fourfold anisotropy field was observed ( $\sim 540$ Oe to $\sim 710$ Oe from room temperature to $24 \mathrm{~K}$ ).

\begin{tabular}{|c|c|c|c|c|c|}
\hline & \multirow[b]{2}{*}{ Sample } & \multicolumn{2}{|c|}{ RT } & \multicolumn{2}{|c|}{$24 \mathrm{~K}$} \\
\hline & & $\begin{array}{l}4 \pi M_{e f f} \\
(\mathrm{kOe})\end{array}$ & $\begin{array}{c}H ॥ \\
(\mathrm{kOe})\end{array}$ & $\begin{array}{l}4 \pi M_{e f f} \\
(\mathrm{kOe})\end{array}$ & $\begin{array}{c}H ॥ \\
(\mathrm{kOe})\end{array}$ \\
\hline \multirow{2}{*}{ 藏 } & $\mathrm{Fe}(2.59 \mathrm{~nm})$ & 15.85 & 0.41 & 16.17 & 0.61 \\
\hline & $\mathrm{Fe}(5.19 \mathrm{~nm})$ & 18.91 & 0.53 & 18.65 & 0.68 \\
\hline \multirow{3}{*}{$\frac{\infty}{\stackrel{0}{0}}$} & $\mathrm{Fe}(2.60 \mathrm{~nm}) / \mathrm{FeF}_{2}(50 \mathrm{~nm})$ & 14.15 & 0.44 & 12.88 & 0.68 \\
\hline & $\mathrm{Fe}(2.62 \mathrm{~nm}) / \mathrm{KNiF}_{3}(16 \mathrm{~nm})$ & 14.98 & 0.45 & 14.04 & 0.67 \\
\hline & $\mathrm{Fe}(2.62 \mathrm{~nm}) / \mathrm{KNiF}_{3}(50 \mathrm{~nm})$ & 15.17 & 0.43 & 14.50 & 0.69 \\
\hline \multirow{13}{*}{ 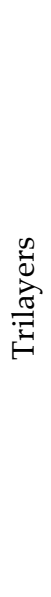 } & $\mathrm{Fe}(2.60 \mathrm{~nm}) / \mathrm{KNiF}_{3}(0.8 \mathrm{~nm}) / \mathrm{FeF}_{2}(50 \mathrm{~nm})$ & 14.18 & 0.43 & 12.94 & 0.65 \\
\hline & $\mathrm{Fe}(2.61 \mathrm{~nm}) / \mathrm{KNiF}_{3}(1.2 \mathrm{~nm}) / \mathrm{FeF}_{2}$ & 15.92 & 0.48 & 14.79 & 0.71 \\
\hline & $\mathrm{Fe}(2.60 \mathrm{~nm}) / \mathrm{KNiF}_{3}(1.7 \mathrm{~nm}) / \mathrm{FeF}_{2}$ & 14.44 & 0.47 & 13.15 & 0.76 \\
\hline & $\mathrm{Fe}(2.58 \mathrm{~nm}) / \mathrm{KNiF}_{3}(2.0 \mathrm{~nm}) / \mathrm{FeF}_{2}$ & 15.62 & 0.43 & 15.15 & 0.645 \\
\hline & $\mathrm{Fe}(2.59 \mathrm{~nm}) / \mathrm{KNiF}_{3}(2.8 \mathrm{~nm}) / \mathrm{FeF}_{2}$ & 14.76 & 0.45 & 13.84 & 0.66 \\
\hline & $\mathrm{Fe}(2.59 \mathrm{~nm}) / \mathrm{KNiF}_{3}(3.6 \mathrm{~nm}) / \mathrm{FeF}_{2}$ & 14.90 & 0.46 & 13.76 & 0.68 \\
\hline & $\mathrm{Fe}(2.68 \mathrm{~nm}) / \mathrm{KNiF}_{3}(4.0 \mathrm{~nm}) / \mathrm{FeF}_{2}$ & 15.62 & 0.42 & 15.52 & 0.63 \\
\hline & $\mathrm{Fe}(2.61 \mathrm{~nm}) / \mathrm{KNiF}_{3}(5.2 \mathrm{~nm}) / \mathrm{FeF}_{2}$ & 15.51 & 0.45 & 14.08 & 0.77 \\
\hline & $\mathrm{Fe}(2.60 \mathrm{~nm}) / \mathrm{KNiF}_{3}(6.0 \mathrm{~nm}) / \mathrm{FeF}_{2}$ & 15.00 & 0.49 & 13.37 & 0.77 \\
\hline & $\mathrm{Fe}(2.58 \mathrm{~nm}) / \mathrm{KNiF}_{3}(91.2 \mathrm{~nm}) / \mathrm{FeF}_{2}$ & 17.27 & 0.43 & 14.89 & 0.64 \\
\hline & $\mathrm{Fe}(5.29 \mathrm{~nm}) / \mathrm{KNiF}_{3}(0.8 \mathrm{~nm}) / \mathrm{FeF}_{2}(50 \mathrm{~nm})$ & 19.22 & 0.54 & 19.095 & 0.73 \\
\hline & $\mathrm{Fe}(5.29 \mathrm{~nm}) / \mathrm{KNiF}_{3}(2.8 \mathrm{~nm}) / \mathrm{FeF}_{2}$ & 19.11 & 0.55 & 18.99 & 0.75 \\
\hline & $\mathrm{Fe}(5.15 \mathrm{~nm}) / \mathrm{KNiF}_{3}(4.0 \mathrm{~nm}) / \mathrm{FeF}_{2}$ & 18.96 & 0.53 & 19.07 & 0.68 \\
\hline
\end{tabular}

Table 1. Values of the effective demagnetizing field $4 \pi \mathrm{Meff}( \pm 0.06 \mathrm{kOe})$ and the fourfold iron anisotropy field $\mathrm{H} ॥( \pm 0.01 \mathrm{kOe})$ magnitudes at $24 \mathrm{~K}$ from linear fitting extrapolations for trilayer systems of $\mathrm{Fe} / \mathrm{KNiF}_{3} / \mathrm{FeF}_{2}(50 \mathrm{~nm})$ in comparison to single Fe layer and bilayers $\mathrm{Fe} / \mathrm{FeF}_{2}, \mathrm{Fe} / \mathrm{KNiF}_{3}$. 
The effective demagnetizing field $\left(4 \pi M_{\text {eff }}\right)$ value is less than $21.4 \mathrm{kOe}$ at room temperature. A possible explanation for this is the existence of surface anisotropy and uniaxial anisotropy fields, $H_{s}$ and $H_{u}$ respectively, such that $4 \pi M_{e f f}=4 \pi M s-H_{u}-H_{s}$. It is interesting to note that the $4 \pi M_{\text {eff }}$ for the thinner Fe layers decreases slightly with decreasing temperature from 15.1 $\mathrm{kG}$ to $\sim 14.1 \mathrm{kG}$ between room temperature and $24 \mathrm{~K}$. The thicker Fe layers show $4 \pi M_{\text {eff }}$ nearly constant $(\sim 19.0 \mathrm{kG})$ between room temperature and $24 \mathrm{~K}$. This is suggestive of an out of plane anisotropy induced by the $\mathrm{KNiF}_{3}$ interface.

\section{1. $\mathrm{Fe} / \mathrm{FeF}{ }_{2}$ and $\mathrm{Fe} / \mathrm{KNiF}_{3}$ reference structures}

The resonance field for the reference bilayers is less than that of the single Fe film data. This reduction is attributed to the AFM-induced dynamic anisotropy field $H_{d y n}^{A F M}$. The temperature dependence of the resonance field for easy and hard axis field orientation for bilayer $\mathrm{Fe} / \mathrm{FeF}_{2}$ is given in figure 2(a) and the inset shows the experimental results for the single Fe film. In figure 2(b) and (c) the temperature dependence of $H_{d y n}^{A F M}$ for easy and hard axis field orientation for reference bilayers $\mathrm{Fe} / \mathrm{FeF}_{2}$ and $\mathrm{Fe} / \mathrm{KNiF}_{3}$ is presented. The values of $H_{d y n}^{A F M}$ were obtained as the difference between the resonance field data of bilayers and the linear fit extrapolated to low temperature. For each sample the linear fit becomes from the high-temperature (i.e. greater than blocking temperature) resonance field dependence.

The values for the exchange bias $H_{E}$ were obtained from scans with positive and negative applied magnetic fields. According to Eq. (1) the asymmetry between resonance fields at each $180^{\circ}$ interval is $2 H_{E}$. For $\mathrm{Fe}(2.60 \mathrm{~nm}) / \mathrm{FeF}_{2}(50.1 \mathrm{~nm})$ structure value of $H_{E}$ at $24 \mathrm{~K}$ was found to be 185 Oe and is significally smaller (by a factor of four) than $H_{d y n}^{A F M}$ determined for this sample (see figure2(b)). At the same time, the FMR results show a low value of $H_{d y n}^{A F M}$ for $\mathrm{Fe}(2.62 \mathrm{~nm}) / \mathrm{KNiF}_{3}\left(16.0 \mathrm{~nm}\right.$ ) bilayer (see figure2(c)). An exchange bias field of $H_{E}=19$ Oe was found, in agreement with the value reported by Wee et al.[8, 9].

\section{2. $\mathrm{Fe} / \mathrm{KNiF}_{3} / \mathrm{FeF}_{2}$}

An example of how $H_{d y n}^{A F M}$ varies with temperature for trilayers is shown in figure 3. Values extracted for $H_{d y n}^{A F M}$ display several interesting features. The first is that $H_{d y n}^{A F M}$ is a nonmonotonic function of field orientation. Figure 3 shows the temperature dependence of the $H_{d y n}^{A F M}$ for $\mathrm{Fe}(2.6 \mathrm{~nm}) / \mathrm{KNiF}_{3}(0.8 \mathrm{~nm}) / \mathrm{FeF}_{2}(50 \mathrm{~nm})$ structure for three different orientations of the applied field: along an easy axis (EA), a hard axis (HA) and midway between (IA; $\left.\phi_{H}=22.5^{\circ}\right)$. Values of $H_{d y n}^{A F M}$ are similar for hard and intermediate axis orientations and are significantly larger than that determined for easy axis orientation (at 24K: 1040 Oe, 1048 Oe and 757 Oe, respectively). 


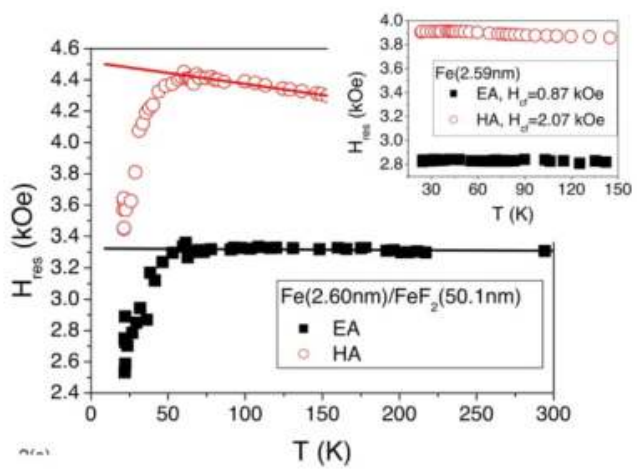

(a)

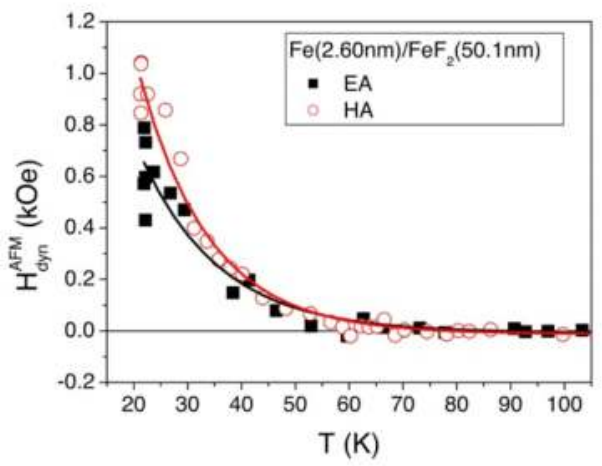

(b)

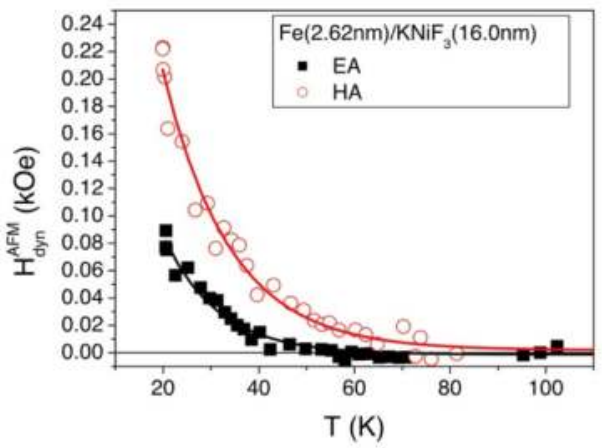

(c)

Figure 2. (a) Temperature dependence of the resonance field for bilayer $\mathrm{Fe}(2.60 \mathrm{~nm}) / \mathrm{FeF}_{2}(50.1 \mathrm{~nm})$. The inset in (a) shows the linear temperature dependence of the reference Fe layer, (b) Temperature dependence of $H_{d y n}^{A F M}$ for bilayer $\mathrm{Fe} / \mathrm{FeF}_{2}$ and (c) $\mathrm{Fe} / \mathrm{KNiF}_{3}$. Filled squares denote measurements along Fe easy axis $\left(\mathrm{EA} ; \varphi_{\mathrm{H}}=45^{\circ}\right)$ and unfilled circles along Fe hard axis $\left(\mathrm{HA} ; \varphi_{\mathrm{H}}=0^{0}\right)$. The cooling field was $\mathrm{H}_{\mathrm{cf}}$ $=0.87 \mathrm{kOe}$ and $\mathrm{H}_{\mathrm{cf}}=2.07 \mathrm{kOe}$ for Fe easy and hard axis, respectively. The lines are guides to the eye. 


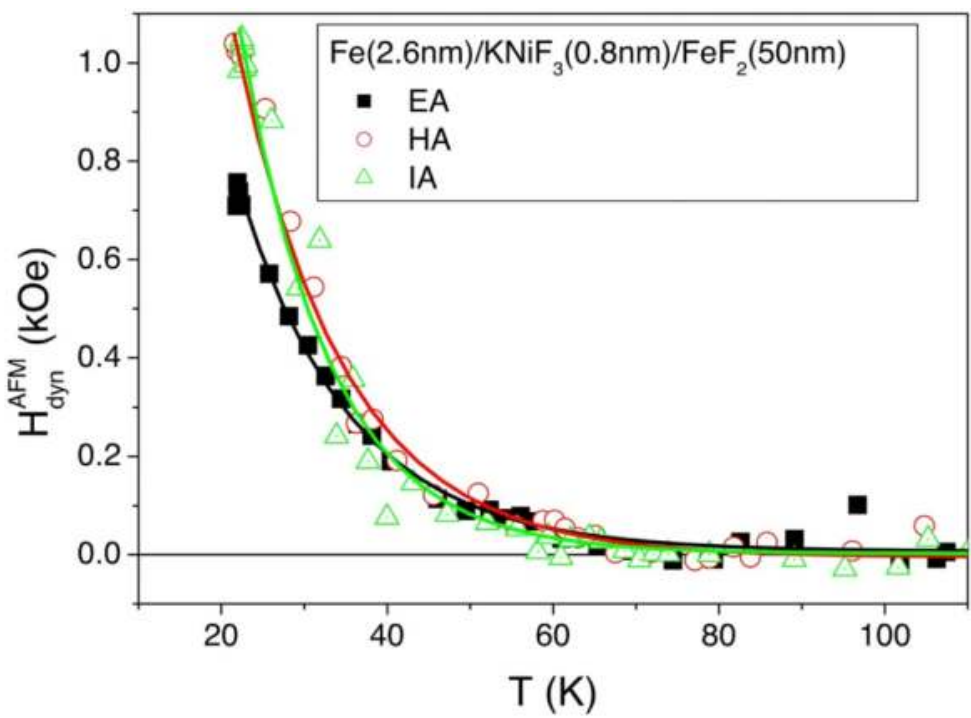

Figure 3. Temperature dependence of the $H_{d y n}^{A F M}$ for trilayer structure $\mathrm{Fe}(2.6 \mathrm{~nm}) / \mathrm{KNiF}_{3}(0.8$ $\mathrm{nm}) / \mathrm{FeF}_{2}(50 \mathrm{~nm})$ for three different orientations of the applied field: along an easy axis (EA), hard axis (HA) and midway between (IA; $\left.\phi \mathrm{H}=22.5^{\circ}\right)$.

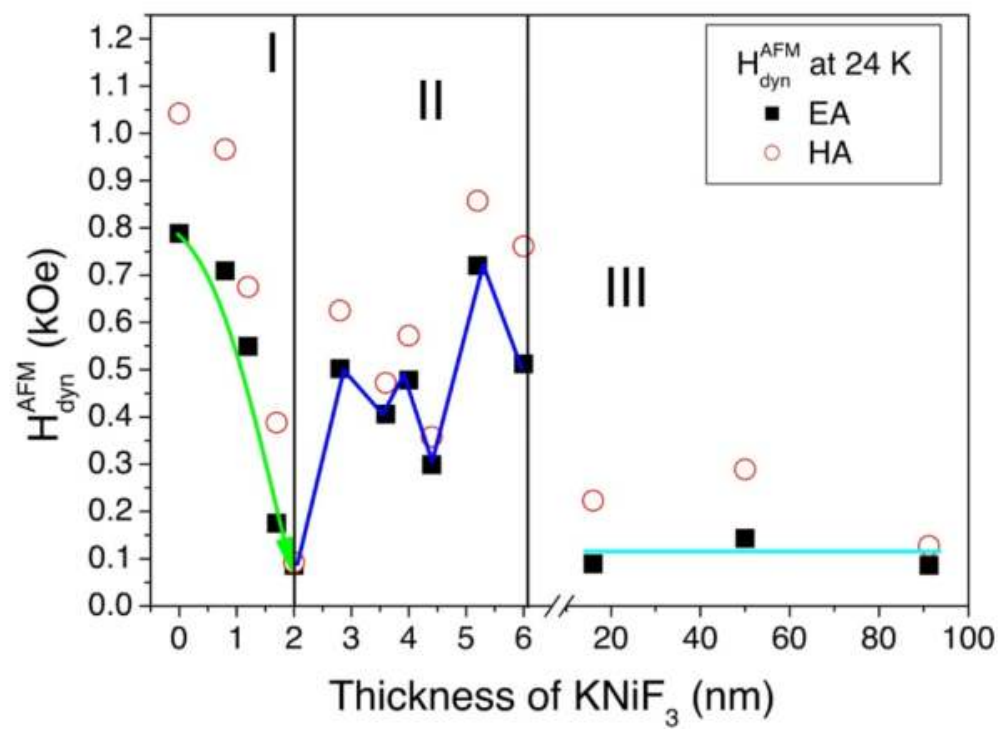

Figure 4. $H_{d y n}^{A F M}$ as a function of the $\mathrm{KNiF}_{3}$ film thickness at $24 \mathrm{~K}$ for structures with a $2.6 \mathrm{~nm}$-thick Fe layer. 
Another interesting feature is associated with the dependence of $H_{d y n}^{A F M}$ on $\mathrm{KNiF}_{3}$ thickness. In figure 4 we show $H_{d y n}^{A F M}$ as a function of the $\mathrm{KNiF}_{3}$ film thickness at $24 \mathrm{~K}$ for structures with a $2.6 \mathrm{~nm}$-thick Fe layer. Note that the value of this anisotropy initially decreases very rapidly to almost zero for the $2 \mathrm{~nm}$-thick $\mathrm{KNiF}_{3}$ layer. For samples with a thicker $\mathrm{KNiF}_{3}$ layers $\left(>2 \mathrm{~nm}\right.$ ), the value of $H_{d y n}^{A F M}$ increases and exhibits a non-monotonic dependence on thickness. For a fairly thick $\mathrm{KNiF}_{3}$ layer $(91.2 \mathrm{~nm})$ the value of the AFM-induced dynamic anisotropy reaches saturation at approximately 100 Oe. Note that even in this regime a difference of 40 Oe persists for values with the field measured along easy and hard axes.

We also mention results from field cooling. It has repeatedly been shown that the value of the unidirectional anisotropy $\mathrm{He}$ depends upon the cooling field strength. A stronger cooling field leads to more pinned spins and larger values for the exchange bias. We have tested the dependence of $H_{d y n}^{A F M}$ on cooling field strength and determined that there is no such dependence in fields up to $1 \mathrm{~T}$. Instead, the magnitude of $H_{d y n}^{A F M}$ for different orientations probably depends on magnitude of the AFM anisotropy field.

Finally, we demonstrate that $H_{d y n}^{A F M}$ depends on the $\mathrm{KNiF}_{3}$ thickness. Values of $H_{d y n}^{A F M}$ are tabulated in table 2 for two different thicknesses of $\mathrm{Fe}$ in $\mathrm{Fe} / \mathrm{KNiF}_{3} / \mathrm{FeF}_{2}$.

\begin{tabular}{lllllll}
\hline \hline & $H_{d y n}^{A F M}\left(\mathrm{t}_{\mathrm{Fe}}=2.6 \mathrm{~nm}\right)$ & $H_{d y n}^{A F M}\left(\mathrm{t}_{\mathrm{Fe}}=5.29 \mathrm{~nm}\right)$ & \multicolumn{2}{l}{$\frac{H_{d y n}^{A F M}(\text { thin } F e)}{H_{d y n}^{A F M}(\text { thick Fe })}$} \\
\cline { 2 - 7 } & $\mathrm{EA} \mathrm{(Oe)}$ & $\mathrm{HA}(\mathrm{Oe})$ & $\mathrm{EA} \mathrm{(Oe)}$ & $\mathrm{HA}(\mathrm{Oe})$ & along EA & along HA \\
$\mathrm{Fe} / \mathrm{KNiF}_{3}(0.8 \mathrm{~nm}) / \mathrm{FeF}_{2}$ & 706 & 966 & 301 & 429 & 2.34 & 2.25 \\
$\mathrm{Fe} / \mathrm{KNiF}_{3}(2.8 \mathrm{~nm}) / \mathrm{FeF}_{2}$ & 502 & 625 & 244 & 333 & 2.06 & 1.88 \\
\hline \hline
\end{tabular}

Table 2. Comparison of the $H_{d y n}^{A F M}$ values obtained for trilayer structures with two different thicknesses of FM layer.

Doubling the thickness of the Fe reduces $H_{d y n}^{A F M}$ by approximately a factor of two for each $\mathrm{KNiF}_{3}$ thicknesses, as one would expect for an interface effect. We therefore conclude that the results displayed in figure 4 may be related to spin structure within the $\mathrm{KNiF}_{3}$.

\subsection{FMR linewidth}

Ferromagnetic resonance linewidth is the primary parameter, outside resonance field, considered in the experiment. In Figure $5(\mathrm{a}, \mathrm{b}, \mathrm{c})$ is shown FMR linewidth $\Delta \mathrm{H}$ as function of temperature for selected reference $\mathrm{Fe} / \mathrm{FeF}_{2}$ and $\mathrm{Fe} / \mathrm{KNiF}_{3}$ samples and $\mathrm{Fe} / \mathrm{KNiF}_{3} / \mathrm{FeF}_{2}$ trilayers.

The FMR linewidth for the single Fe layer displays a linear dependence on temperature. On the contrary, the observed temperature changes in linewidth of the exchange coupled systems are strong and non-linear. As an instance, $\Delta H$ of $\mathrm{Fe}(2.60 \mathrm{~nm}) / \mathrm{KNiF}_{3}(0.8 \mathrm{~nm})$ 
$/ \mathrm{FeF}_{2}(50.1 \mathrm{~nm})$ increases 12 times with decreasing temperature to $24 \mathrm{~K}$ when the magnetic field was applied along an easy axis. When the FMR measurements were carried out with the field applied along hard axis, the observed increment is eightfold.

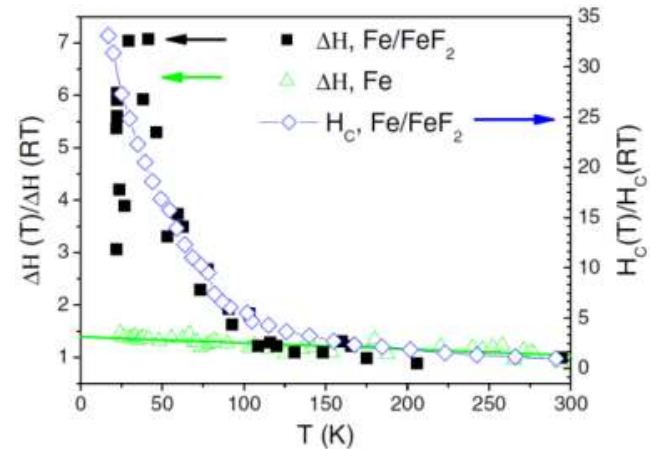

(a)

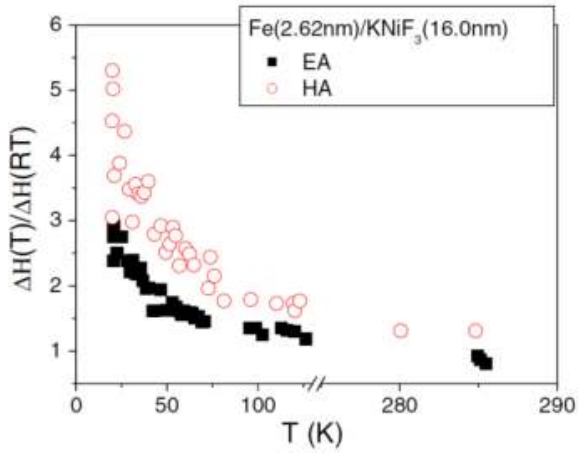

(b)

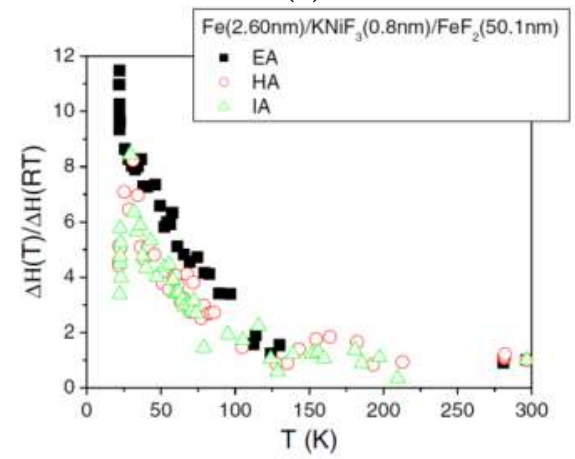

(c)

Figure 5. (a) Ferromagnetic resonance linewidths $\Delta \mathrm{H}$ for $\mathrm{Fe} / \mathrm{FeF}$. For comparison, the temperature dependence of $\Delta \mathrm{H}$ for single $\mathrm{Fe}$ is shown. Temperature dependence of coercive field $\mathrm{Hc}$ for $\mathrm{Fe} / \mathrm{FeF}_{2}$ is presented on the right-hand side scale. (b) Ferromagnetic resonance linewidths $\Delta \mathrm{H}$ for $\mathrm{Fe} / \mathrm{KNiF}_{3}$ and (c) trilayer $\mathrm{Fe} / \mathrm{KNiF}_{3} / \mathrm{FeF}_{2}$ as functions of temperature. 
We proposed on explanation for FMR linewidth behavior that depends upon of inhomogeneity existing in the local fields acting on the ferromagnet. Some evidence for this may come from our MOKE magnetometer studies on $\mathrm{Fe} / \mathrm{FeF}_{2}$ structure [23] and the observations made by some of us for $\mathrm{Fe} / \mathrm{KNiF}_{3}$ system $[8,9]$. For these reference bilayers a close correspondence between linewidth and coercive field $H_{c}$ (see figure 5(a)) was noted. Similarly to FMR linewidth theory, the spatial inhomogeneities in the local internal fields can have a large effect on the coercive fields and magnetization loop widths.

\section{Discussion}

The temperature dependence of $H_{d y n}^{A F M}$ described above behaves generally like that observed previously for $\mathrm{Fe} / \mathrm{KNiF}_{3}$ bilayers. A rotatable anisotropy was able to describe well FMR results in the previous work. Possible mechanisms underlying the formation of the rotatable anisotropy were discussed in references [8] and [9], and argued to be consistent with measured linewidths. The essential idea is that the Fe exchange couples to different regions of the $\mathrm{KNiF}_{3}$ interface, and coupling between these regions can produce a rotatable anisotropy and coercivity as observed experimentally.

Similar arguments should apply in the present case. In the case of trilayers additional measurements were made in relation to reference bilayers, namely for $\mathrm{Fe} / \mathrm{KNiF}_{3}(0.8 \mathrm{~nm}) / \mathrm{FeF}_{2}$ sample temperature dependence of the $H_{d y n}^{A F M}$ for three field orientations (EA, HA, IA) was studied. It turned out that the $H_{d y n}^{A F M}$ is a non-monotonic function of the field orientation. We have at present no evidence to suggest a mechanism for this anisotropy, but can speculate that the presence of the second interface with high anisotropy $\mathrm{FeF}_{2}$ introduces an additional competition that may affect alignment of regions in the $\mathrm{KNiF}_{3}$.

It seems that a lot of information to verify the introduced model of $H_{d y n}^{A F M}$ could be obtained from studies of Fe/AFM1/AFM2 with single crystalline AFMs. However, the studies of Fe/AFM bilayers, where AFM was deposited onto single crystal of $\mathrm{Fe}$, either in the polycrystalline or single crystal form do not give a clear picture. The systematic study of the influence of in-plane crystalline quality of the antiferromagnet on anisotropies in $\mathrm{Fe} / \mathrm{FeF}_{2}$ structure were examined by Fitzsimmons et al [22]. In this study three types of samples were investigated with polycrystalline ferromagnetic Fe thin films and antiferromagnetic $\mathrm{FeF}_{2}$ as: (i) untwinned single crystal; (ii) twinned single crystal, and (iii) textured polycrystal. The results obtained suggest that the value of exchange bias depends on many conditions, such as: the orientation between the spins in AFM and FM during field cooling; the choice of cooling field orientation relative to the AFM; engineering the AFM microstructure and so on. The bilayer Fe/AFM structures consisting of single-crystal $\mathrm{Fe}$ and $\mathrm{KNiF}_{3}$, or $\mathrm{KCoF}_{3}$, or $\mathrm{KFeF}_{3}$ films were investigated by some of us $[8,9,24,25]$. All antiferromagnets in the samples had single crystalline structure or polycrystalline one with a high degree of texture. The crystalline quality of the antiferromagnets significantly affects the size of training effects, the magnitude 
of the parameters such as: FMR linewidth, the blocking temperature, the exchange bias. $\Delta H s$ of the samples with the single crystal fluorides were reduced compared to the polycrystalline structures. At the same time, the observed changes of the blocking temperature, the exchange bias, the coercivity vs the crystallity of the antiferromagnet film were different for different fluorides. It therefore seems indisputable that only the FMR linewidth is a parameter that uniquely altered depending on poly- or single crystalline nature of AFMs.

Perhaps the most curious feature observed was the complex dependence of $H_{d y n}^{A F M}$ on $\mathrm{KNiF}_{3}$ thickness as illustrated in figure 4 . Three different behaviors were observed, corresponding to three thickness regions: 0 to $2 \mathrm{~nm}, 2$ to $6 \mathrm{~nm}$ and everything greater than $6 \mathrm{~nm}$. The first two regions involve only a few monolayers of $\mathrm{KNiF}_{3}$. Some insight into reasons underlying the existence of these three regions can be obtained by considering possible spin configurations in a model trilayer. The reason is that $\mathrm{KNiF}_{3}$ is a weak antiferromagnet, and so we might expect that strong interlayer coupling to the $\mathrm{Fe}$ and $\mathrm{FeF}_{2}$ might result in large modifications of the spin ordering near the interfaces. Characteristic length scales would correspond to a few nanometers. We explore this idea in some detail with a model described in the appendix. The model makes use of an iterative energy minimization scheme and was used to examine static equilibrium configurations of spins in a thin film trilayer using material parameters appropriate to the $\mathrm{Fe} / \mathrm{KNiF}_{3} / \mathrm{FeF}_{2}$ trilayers studied in this work. Details of the model are left for the appendix, and we discuss below only the essential results and implications for understanding the dependence of $H_{d y n}^{A F M}$ on $\mathrm{KNiF}_{3}$ thickness.

According to our calculations, for example for trilayer structure $\mathrm{Fe} / \mathrm{KNiF}_{3}(3 \mathrm{ML}) / \mathrm{FeF}_{2}$, we find that for angles $\theta_{H}$ (the direction of the applied field is characterized by the angle $\theta_{H}$ ) from $0^{0}$ to $-95^{0}$ the net moments in the first layers of both AFM's oppose the direction of the applied field. Between $-95^{0}$ and $-96^{0}$ a sudden change in spin orientation appears in the first layer of the $\mathrm{FeF}_{2}$. In order to understand how these results may illuminate our measured results for $H_{d y n}^{A F M}$, we note first that the canted spin configuration calculated for all $\mathrm{KNiF}_{3}$ thicknesses is suggestive of Koon's model [26] for exchange bias. In this model, a net moment at the interface is created by spin canting at a compensated interface of the antiferromagnet in contact with the ferromagnet. It was later pointed out by Schulthess and Butler [27] and discussed by Stiles and McMichael [28] that this canting was not itself sufficient to produce bias due to instabilities in the canted configuration. The instabilities allow the canted moment to reverse into a configuration whose energy is the same as the original field cooled orientation energy.

The same principle applies in our case for the largest thickness $\mathrm{KNiF}_{3}$ films. However for small $\mathrm{KNiF}_{3}$ thicknesses, the energy of the reversed state is not the same as for the field cooled orientation. The difference in energies for the $\theta_{H}=0$ and $\theta_{H}=180^{\circ}$ configurations can be sizable, and is shown in figure 6 as a function of $\mathrm{KNiF}_{3}$ thickness where $1 \mathrm{ML}=\mathrm{a}\left(\mathrm{KNiF}_{3}\right)=0.4013 \mathrm{~nm}$ (Muller et al. [10]). 


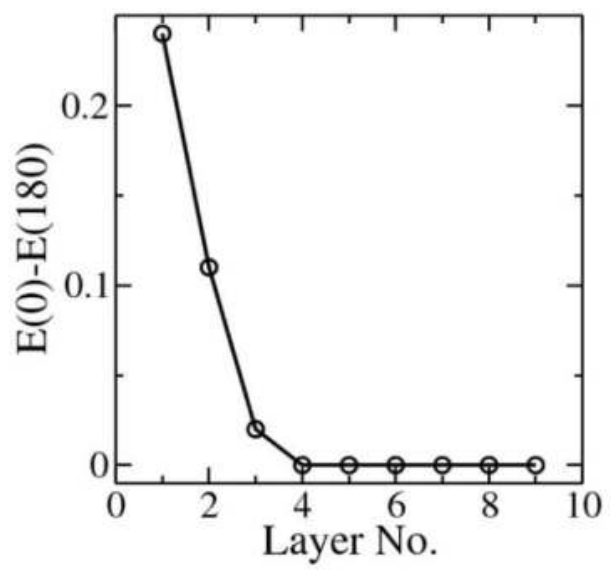

Figure 6. The difference in energies for the $\theta_{\mathrm{H}}=0^{\circ}$ and $\theta_{\mathrm{H}}=180^{\circ}$ configurations as a function of $\mathrm{KNiF}_{3}$ thickness, where $1 \mathrm{ML}=\mathrm{a}\left(\mathrm{KNiF}_{3}\right)=0.4013 \mathrm{~nm}$ (Muller et al.[10]).

This asymmetry in energies disappears when the $\mathrm{KNiF}_{3}$ is thicker than $4 \mathrm{ML}$. This thickness is characteristic of the depth over which a twist can develop in the $\mathrm{KNiF}_{3}$. Above $4 \mathrm{ML}$, the twist can extend to $180^{\circ}$, and behave like the 'partial' wall in the Stiles and McMichael picture of exchange bias[28, 29].

On the basis of this, we suggest that $H_{d y n}^{A F M}$ is associated with the gradient of the energy as a function of $\theta_{\mathrm{H}}$, and that this energy is different for small and large $\theta_{\mathrm{H}}$ for $\mathrm{KNiF}_{3}$ thicknesses less than a domain wall width. $\mathrm{KNiF}_{3}$ thicknesses sufficiently larger than this support formation of a partial wall that effectively isolates the Fe from the $\mathrm{KNiF}_{3}$ interface. This results in an energy whose gradient is the same for 0 and $180^{\circ}$ orientation of the field. As such, the $H_{d y n}^{A F M}$ arises in this model as a kind of susceptibility of the spin configuration to the orientation of the Fe magnetization (which is controlled by the external applied field).

In this model for $H_{d y n}^{A F M}$, the thinnest $\mathrm{KNiF}_{3}$ thickness region creates asymmetry in energies at 0 and $180^{\circ}$ because the $\mathrm{KNiF}_{3}$ is not wide enough to support a complete partial wall. In this region the effect of $\mathrm{FeF}_{2}$ is apparent. The thickest $\mathrm{KNiF}_{3}$ films support a complete partial wall, and the resulting $H_{d y n}^{A F M}$ are associated with energy gradients that are symmetric with respect to reversal of the applied field. The intermediate thickness region allows spin configurations that are strongly thickness dependent with strongly distorted partial walls. The $H_{d y n}^{A F M}$ in this region vary strongly with $\mathrm{KNiF}_{3}$ thickness, in a manner determined by details of configurational energies of the distorted partial wall. Lastly, we note that this picture can also explain the dependence of $H_{d y n}^{A F M}$ on field orientation with respect to easy and hard directions. This follows because the magnitude of $H_{d y n}^{A F M}$ will depend on orientation of the Fe with respect to anisotropy symmetry axes. 


\section{Conclusions}

In summary, we show that a ferromagnet exchange coupled to an antiferromagnetic bilayer can allow the character and strength of exchange anisotropy to be modified. We have studied using FMR exchange bias and exchange anisotropy for Fe exchange coupled to $\mathrm{KNiF}_{3} / \mathrm{FeF}_{2}$. The trilayer was grown by MBE. The temperature dependence of the ferromagnetic resonance peak position shows a characteristic negative shift of the resonance from that of a single Fe layer. This negative shift is a direct result of the exchange coupling between ferromagnetic and antiferromagnetic layers and results in a dynamically induced field $H_{d y n}^{A F M}$.

The value of $H_{d y n}^{A F M}$ is different for measurements performed along ferromagnet easy and hard axes for all the bilayer and trilayer samples. The largest values were found for the field along Fe hard axis. The dependence of the $H_{d y n}^{A F M}$ on the thickness of the $\mathrm{KNiF}_{3}$ is nonmonotonic, reaching a minimum for $2.0 \mathrm{~nm}$ thick $\mathrm{KNiF}_{3}$. For structures with thinner $\mathrm{KNiF}_{3}$, the magnitude of the $H_{d y n}^{A F M}$ increases with decreasing thickness, reaching the maximum expected for the bilayer $\mathrm{Fe} / \mathrm{FeF}_{2}$.

Results from a calculational model for the equilibrium configuration of spins in a ferromagnet/antiferromagnet/antiferromagn trilayer suggests that a form of spin canting may occur at the antiferromagnetic interfaces. For sufficiently thin $\mathrm{KNiF}_{3}$, significant spin canting at the $\mathrm{Fe}$ and $\mathrm{FeF}_{2}$ interfaces occurs due to exchange coupling. Effects of the $\mathrm{FeF}_{2}$ are effectively isolated by $\mathrm{KNiF}_{3}$ thick enough to support a partial magnetic domain wall. As a general result, we suggest that $H_{d y n}^{A F M}$ is a measure of the susceptibility of the interface spin ordering to interface coupling in the $\mathrm{KNiF}_{3}$.

\section{Appendix}

A numerical mean field model for equilibrium spin orientations in a ferromagnet/ antiferromagnet/antiferromagnet is described in this appendix. The spins are treated as classical vectors on a lattice.

The energy of a spin configuration is determined in the following way. An atomistic approach is employed in a mean field approximation. A cubic lattice of vector spins is considered consisting of $\mathrm{N}$ layers representing the first antiferromagnet (called AFM1 and representing the $\mathrm{KNiF}_{3}$ ) and $\mathrm{M}$ layers representing a second antiferromagnet (called AFM2 and representing the $\mathrm{FeF}_{2}$ ). The outermost layer of AFM1 is in contact with a block spin representing the FM. Each layer is described by a unit cell of spins representing the two antiferromagnetic sublattices. Periodic boundary conditions are assumed in the plane of each layer.

The ground state spin configuration is found as follows. The applied magnetic field is set, and a spin site in the FM/AFM1/AFM2 trilayer is chosen. The algorithm is begun by picking a set of values for the initial configuration of spins in each layer corresponding to a field cooled orientation. Exchange coupling between spins is taken into account in addition to 
anisotropy energies, so that the energy of the spins in any given layer depends on the orientation and magnitude of the spins in the nearby layers. The orientation of a spin in layer " $i$ " is characterized by the angle $\theta_{n}$ made with respect to the applied field. A low energy magnetic configuration is obtained when the energy is minimized [30] i.e. the angle $\theta_{n}$ is determined by rotating the spin into the direction of a local effective field calculated from the gradient of the energy at the spin site. The procedure determines the configuration in the following manner: (i) a given layer is randomly chosen and the spins in that layer are rotated to be parallel to the local effective field; (ii) the process is continued until one has a self-consistent, stable state where all the spins are aligned with the local effective fields produced by the neighboring spins.

In this model, the multilayer is treated as an effective one-dimensional system with one spin representing an entire sublattice in a given layer. Only nearest-neighbor exchange coupling is considered. The energy is defined by:

$$
\begin{gathered}
H=H_{A F M}+H_{\mathrm{int}} \\
H_{A F M}=H_{a}^{1,2}+H_{b}^{1,2}
\end{gathered}
$$

where $H_{A F M}$ is the Hamiltonian representing a given antiferromagnet with its both sublattices, and $H_{i n t}$ is the Hamiltonian describing the interfaces contribution.

The geometry is defined in figure 7. The direction normal to the layer planes is $y$. Each $x z$ plane, for a given value of $y$, depicts one layer of an AFM.

Both $\mathrm{AFMs}, \mathrm{KNiF}_{3}$ and $\mathrm{FeF}_{2}$, exhibit uniaxial anisotropy with easy axes in the $+z$ and $-z$ directions. The first $\mathrm{AFM}$ is $\mathrm{KNiF}_{3}$ and is known G-type meaning that the nearest neighbor coupling is antiferromagnetic. The nearest neighbor exchange constants for each AFM1 layer $\left(J_{N N P}\right)$ and between AFM1 layers $\left(J_{N N}\right)$ are defined as negative values. Each layer of the $\mathrm{KNiF}_{3}$ is assumed to be compensated such that both AFM1 sublattices are present within a given layer. The second $\mathrm{AFM}$ is $\mathrm{FeF}_{2}$ and is regarded as completely uncompensated within a given layer. In this case, the $J_{N N P 2}$ exchange coupling constant along $x$ is defined as positive.

The complete Hamiltonian can be written as follows:

$$
\begin{aligned}
H_{a}^{1}=-\mathbf{H}_{a p p} & \cdot \mathbf{S}_{n(A F M 1)}^{a} \\
& +\sum_{m= \pm 1}\left[J_{N N} \mathbf{S}_{n(A F M 1)}^{a} \cdot \mathbf{S}_{(n+m)(A F M 1)}^{b}+4 J_{N N P} \mathbf{S}_{n(A F M 1)}^{a} \cdot \mathbf{S}_{n(A F M 1)}^{b}\right] \\
& -K_{u}\left(\mathbf{S}_{n(A F M 1)}^{a}\right)^{2} \\
H_{b}^{1}=-\mathbf{H}_{a p p} & \cdot \mathbf{S}_{n(A F M 1)}^{b} \\
& +\sum_{m= \pm 1}\left[J_{N N} \mathbf{S}_{n(A F M 1)}^{b} \cdot \mathbf{S}_{(n+m)(A F M 1)}^{b}+4 J_{N N P} \mathbf{S}_{n(A F M 1)}^{b} \cdot \mathbf{S}_{n(A F M 1)}^{a}\right] \\
& -K_{u}\left(\mathbf{S}_{n(\text { AFM1) }}^{b}\right)^{2}
\end{aligned}
$$




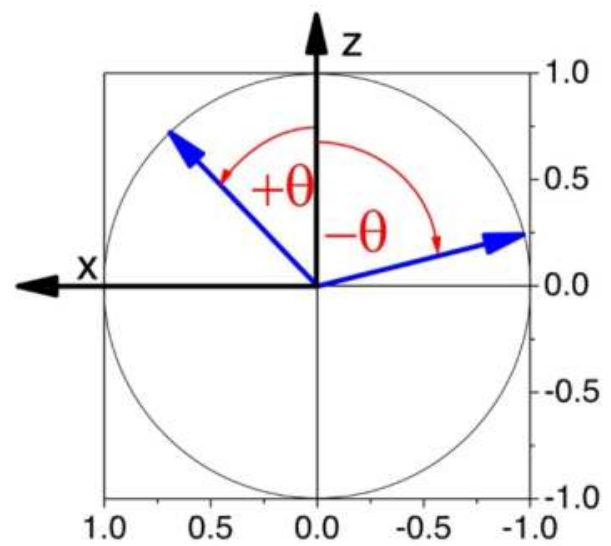

(a)

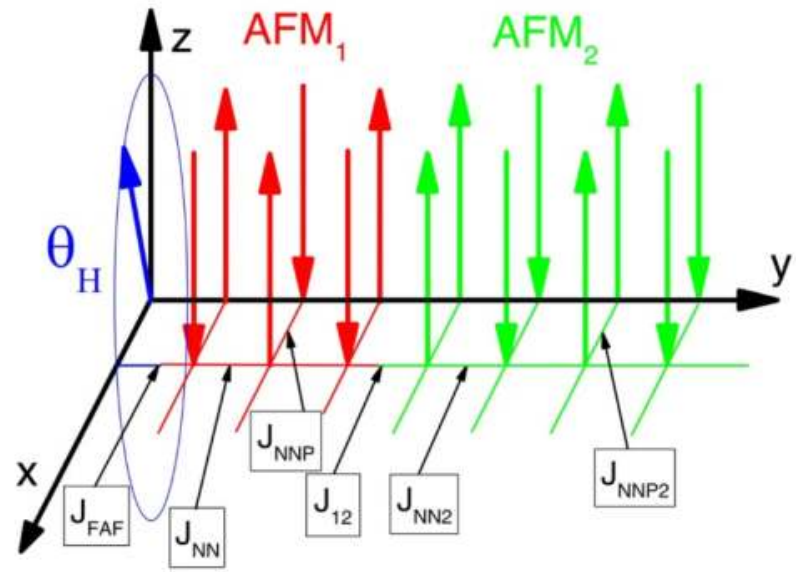

(b)

Figure 7. Geometry of the system (a) defining an angle of the applied field within the film plane $\theta_{\mathrm{H}},(\mathrm{b})$ the direction normal to the layer planes is $y$. Each xz plane, for a given value of $y$, depicts one layer of an AFM.

Similar terms can be written for the second antiferromagnet AFM2. Here, however, a given AFM2 plane is regarded, as completely compensated and only one sublattice is present in the interface:

$$
\begin{aligned}
H_{a}^{2}=-\mathbf{H}_{a p p} & \cdot \mathbf{S}_{n(A F M 2)}^{a} \\
& +\sum_{m= \pm 1} J_{N N 2} \mathbf{S}_{n(A F M 2)}^{a} \cdot \mathbf{S}_{(n+m)(A F M 2)}^{b}+\sum_{n^{\prime}} J_{N N 2 P} \mathbf{S}_{n(A F M 2)}^{a} \cdot \mathbf{S}_{n^{\prime}(A F M 2)}^{a} \\
& -K_{u 2}\left(\mathbf{S}_{n(A F M 2)}^{a}\right)^{2}
\end{aligned}
$$




$$
\begin{aligned}
H_{b}^{2}=-\mathbf{H}_{a p p} & \cdot \mathbf{S}_{n(A F M 2)}^{b} \\
& +\sum_{m= \pm 1} J_{N N 2} \mathbf{S}_{n(A F M 2)}^{b} \cdot \mathbf{S}_{(n+m)(A F M 2)}^{a}+\sum_{n^{\prime}} J_{N N 2 P} \mathbf{S}_{n(A F M 2)}^{b} \cdot \mathbf{S}_{n^{\prime}(A F M 2)}^{b} \\
& -K_{u 2}\left(\mathbf{S}_{n(A F M 2)}^{b}\right)^{2}
\end{aligned}
$$

Finally, the interface term with the FM is given by:

$$
\begin{aligned}
H_{\mathrm{int}}=J_{F A F} \mathbf{S}_{F M} \cdot \mathbf{S}_{n=1(A F M 1)}^{a}-J_{F A F} \mathbf{S}_{F M} \cdot \mathbf{S}_{n=1(A F M 1)}^{b} \\
\quad+J_{12} \mathbf{S}_{n=\max (A F M 1)}^{a} \cdot \mathbf{S}_{n=1(A F M 2)}^{a}-J_{12} \mathbf{S}_{n=\max (A F M 1)}^{b} \cdot \mathbf{S}_{n=1(A F M 2)}^{a}
\end{aligned}
$$

The notation is defined by:

- $H_{a p p}$ is the applied magnetic field,

- $\quad \mathbf{S}_{n}^{a}, \mathbf{S}_{n}^{b}$ are the spin vectors at layer " $n$ " for sublattice " $a$ " and " $b$ " of the AFM1 and AFM2, respectively. No out-of-plane spin component is considered within this model. The only spin arrangement is to lie within $x z$ plane i.e. $(x, 0, z)$,

- $\quad \mathbf{S}_{n^{\prime}}^{a}, \mathbf{S}_{n^{\prime}}^{b}$ are the spin vectors at layer " $n$ " for sublattice " $a$ " and " $b$ ", respectively of the AFM2. Here, the different spin orientation is allowed within one $n$-th layer but again, no out-of-plane spin component is allowed to appear within this model,

- $\quad K_{u}, K_{u 2}$ are site anisotropies for spins at layer " $n$ " of AFM1 and AFM2, respectively.

The exchange coupling constants are defined as:

- $J_{N N}, J_{N N 2}$ are the exchange interaction constants between spins at " $n$ " and " $n+m$ " layers of AFM1 and AFM2, respectively,

- $\quad J_{N N P,} J_{N N P 2}$ are the exchange interaction constants within $n$-th layer of AFM1 and AFM2, respectively,

- JFAF is the interface exchange coupling constant between the FM layer and the first antiferromagnet,

- $J_{12}$ is the interface exchange coupling constant between the first and the second AFM1/AFM2 layer.

Note that the sign of these exchange interactions has been introduced through the numerical values of the parameters. All the values of anisotropy and exchange coupling are given in field units and are summarized in table 3. All calculations were made for 50 atomic layers in AFM2.

An example arrangement of spins in the AFM1 and AFM2 is depicted in figure A1. Bulk values of the anisotropy and the exchange coupling constants for $\mathrm{KNiF}_{3}$ and $\mathrm{FeF}_{2}$ have been used in all calculations. The interface exchange coupling constant between AFMs $\left(J_{12}\right)$ have been assumed to be a geometrical mean of the exchange coupling constants of the adjacent layers. The interface exchange coupling between FM and AFM1 is defined as $Z J_{N N}$, i.e. the exchange coupling within the first AFM1 multiplied by the number of nearest neighbors of each Fe spin site. 


\begin{tabular}{|c|c|c|c|}
\hline AFM & $\begin{array}{l}\text { Exchange field } \\
\text { (kOe) }\end{array}$ & $\begin{array}{l}\text { Anisotropy field } \\
\qquad(\mathrm{kOe})\end{array}$ & $\frac{\text { anisotropy field }}{\text { exchange field }}$ \\
\hline AFM1: $\mathrm{KNiF}_{3}$ & $H_{e x}=3500^{a}$ & $H_{K u}=0.080^{\mathrm{b}}$ & $2.4 \cdot 10^{-5}$ \\
\hline AFM2: $\mathrm{FeF}_{2}$ & $H_{e x 2}=434^{\mathrm{c}}$ & $H_{K u 2}=149^{c}$ & $0.33^{\mathrm{d}}$ \\
\hline
\end{tabular}

a M.E. Lines, Phys. Rev. 164, 736 (1967).

${ }^{\mathrm{b}}$ H. Yamaguchi, K. Katsumata, M, Hagiwara, M. Tokunaga, H. L. Liu, A Zibold, D. B. Tanner, and Y.J.Wang, Phys. Rev. B 59, 6021 (1999).

c M.L. Silva, A.L. Dantas, and A.S. Carrico, Solid State Commun. 135, 769 (2005).

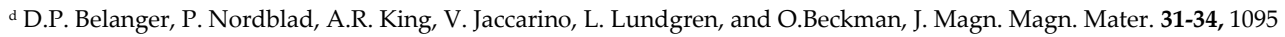
(1983).

Table 3. Used bulk values of the exchange and anisotropy fields for the $\mathrm{KNiF}_{3}$ and $\mathrm{FeF}_{2}$.

After converging to a stable configuration, the total energy per spin site was calculated as a function of the angle of the applied magnetic field, $\theta_{H}$. Example results are shown in figure 8(a) for different thicknesses of the first AFM1.

Note that the horizontal axis $\left(\theta_{H}(\mathrm{deg})\right)$ is the same for all calculated structures, but the total calculated energy is not to scale in order to visibly depict the calculated results for each thickness of AFM1. A discontinuity in the total energy per spin site appears for thicknesses of AFM1 ranging from 1ML to 9ML. The values of angle $\theta_{H}$ for which the discontinuity appears $\left(\theta_{\text {Hdis }}\right)$ is presented in Fig $8(\mathrm{~b})$. Three regions can be distinguished in these results: $1 \mathrm{ML}$ to $4 \mathrm{ML}, 5 \mathrm{ML}$ to $9 \mathrm{ML}$, and $>9 \mathrm{ML}$.

The different regions correspond to different spin arrangements within each AFM layer. An example for the 3ML thick AFM1 is shown in figure 9. The spin arrangements are presented in terms of the angle that each spin vector makes with the $z$-direction. Here, blue arrow represents the applied field, and red arrows are used for the spins in the three layers of AFM1 and green arrows represent the first few spin sites of AFM2.

The comparison between a spin arrangement for $\theta_{H=-95^{0}}$ (top diagrams) and for $\theta_{H=-}=96^{0}$ (bottom diagrams) is shown. The central diagram in figure 9 shows the spin arrangement when the field is applied along $+z$ direction. The first layer of AFM1 experiences spincanting. An energy minimum for the fully compensated interface has been obtained for perpendicular interfacial coupling between the FM and AFM spins. The spin-canting and the resulting net moment induced within the first layer of the AFM1 always opposes the direction of the applied field. Moreover, spin-canting appears also in the second antiferromagnet AFM2 for the first two layers. It is important to note that the direction of the net moment induced in the first layer of AFM2 does not correlate directly to the direction of the applied magnetic field. 


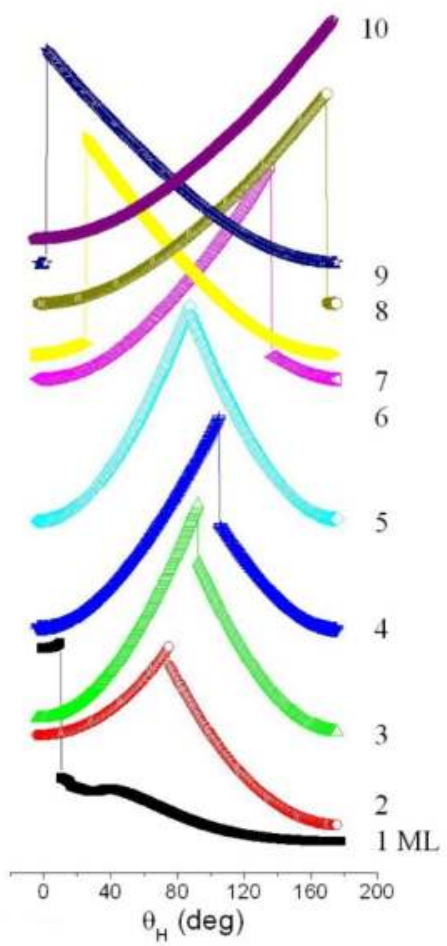

(a)

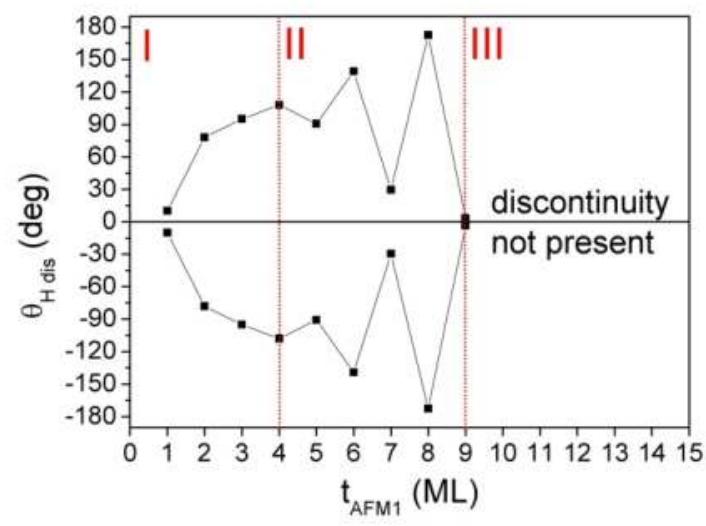

(b)

Figure 8. (a) The total energy per spin site as a function of the angle of the applied magnetic field $\theta_{\mathrm{H}}$ for different thicknesses of the first AFM1. The horizontal axis $\left(\theta_{\mathrm{H}}(\mathrm{deg})\right)$ is the same for all calculated structures, but the total calculated energy is not to scale in order to visibly depict the calculated results for each thickness of AFM1, (b) The values of angle $\theta_{\mathrm{H}}$ for which the discontinuity appears ( $\left.\theta_{\mathrm{Hdis}}\right)$ for thicknesses of AFM1 ranging from 1ML to 9ML. The lines are guides to the eye. 


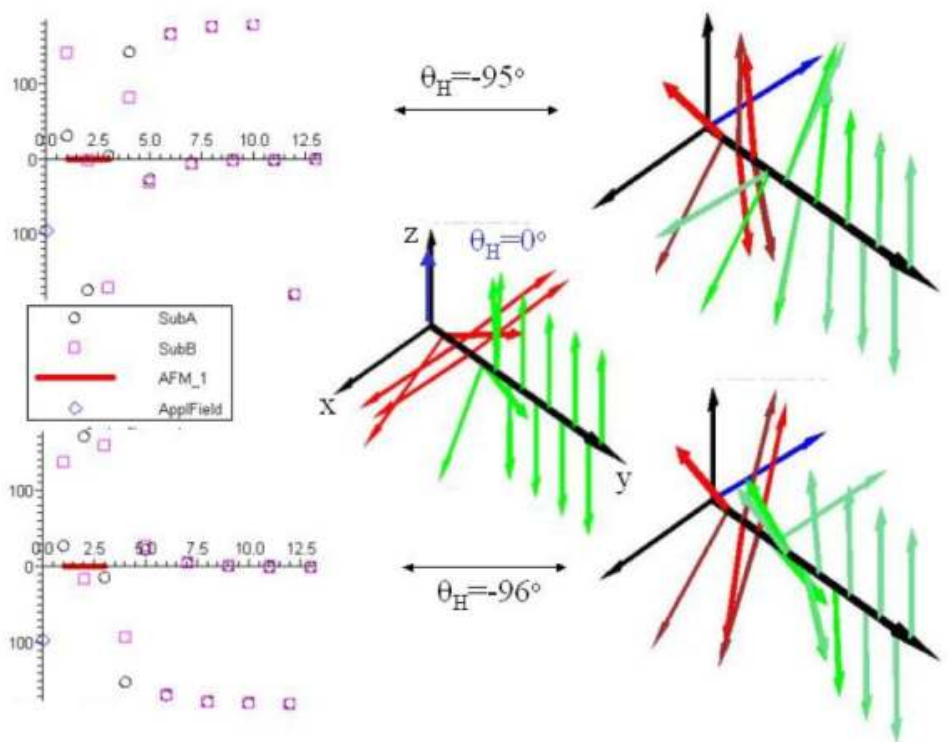

Figure 9. The spin arrangements are presented in terms of the angle that each spin vector makes with the z-direction for the 3ML-thick AFM1. Blue represents the applied field $\mathrm{H}_{\mathrm{a}}$, red arrows are used for the spins in the three layers of AFM1 and green represent the first few spin sites of AFM2.

\section{Author details}

\section{Stefania Widuch}

Center for Magnetism and Magnetic Nanostructures, University of Colorado, Colorado Springs, Colorado, USA

School of Physics (M013), University of Western Australia (UWA), Crawley, WA, Australia Institute of Physics, University of Silesia, Katowice, Poland

Robert L. Stamps

School of Physics and Astronomy, Kelvin Building, University of Glasgow, Scotland, UK

Danuta Skrzypek

Institute of Physics, University of Silesia, Katowice, Poland

\section{Zbigniew Celinski}

Center for Magnetism and Magnetic Nanostructures, University of Colorado, Colorado Springs,

Colorado, USA

\section{Acknowledgement}

RLS acknowledges the Australian Research Council. The work at UCCS was supported by the National Science Foundation Grants (DMR 0605629 and DMR 0907053). SW acknowledges funding through the IEEE for the visit at the UWA Perth. 


\section{References}

[1] J. Nogués, and I. K. Schuller, J. Magn. Magn. Mater. 192, 203 (1999).

[2] A.E. Berkowitz, and K. Takano, J. Magn. Magn. Mater. 200, 552 (1999).

[3] R.L. Stamps, J. Phys. D: Appl. Phys. 33, R247 (2000).

[4] M. Kiwi, J. Magn. Magn. Mater. 234, 584 (2001).

[5] J. Nogués, J. Sort, V. Langlais, V. Skumryev, S. Suriñach, J.S. Muñoz, and M.D. Baró, Phys. Rep. 422, 65 (2005).

[6] A.E. Berkowitz, and R.H. Kodama, in Contemporary Concepts of Condensed Matter Science, editted by D.L. Mills and J.A.C. Bland (Elsevier B.V. 2006), Chapter 5, p. 115.

[7] J. Stöhr, and H.C. Siegmann, Magnetism - From Fundamentals to Nanoscale Dynamics (Springer, New York, 2006).

[8] L. Wee, R.L. Stamps, L. Malkinski, and Z. Celinski, Phys. Rev. B 69, 134426 (2004).

[9] L. Wee, R.L. Stamps, L. Malkinski, Z. Celinski, and D. Skrzypek, Phys. Rev. B 69, 134425 (2004).

[10] O. Muller, and R. Roy, The Major Ternary Structural Families (Springer, NewYorkHeidelberg-Berlin, 1974).

[11] V. Scatturin, L. Corliss, N Elliott, and J. Hastings, Acta Cryst. 14, 19 (1961).

[12] K. Hirakawa, K. Hirakawa, and T. Hashimoto, J. Phys. Soc. Jpn. 15, 2063 (1960).

[13] Z. Celinski, and D. Skrzypek, Acta Phys. Pol. A 65, 149 (1984).

[14] R.A. Erickson, Phys. Rev. 90, 779 (1953).

[15] W. Stout and S.A. Reed, J. Am. Chem. Soc. 76, 5279 (1954).

[16] W. Jauch, A. Palmer and A.J. Schultz, Acta Cryst. B49, 984 (1993); K. Haefner, Ph.D. thesis, Univ. of Chicago, 1964.

[17] M.T. Hutchings, B.D.Rainford, and H.J. Guggenheim, J. Phys. C 3, 307 (1970).

[18] M.R. Fitzsimmons, B.J. Kirby. S. Roy, Zhi-Pan Li, Igor V. Roshchin, S.K. Sinha, and I.K. Schuller, Phys. Rev. B 75, 214412 (2007).

[19] J. Nogués, D. Lederman, T.J. Moran, I.K. Schuller, and K.V. Rao, Appl. Phys. Lett. 68, 3186 (1996).

[20] J. Nogués, T.J. Moran, D. Lederman, and I.K. Schuller, Phys. Rev. B 59, 6984 (1999).

[21] M.R. Fitzsimmons, P. Yashar, C. Leighton, I.K Schuller, J. Nogués, C.F. Majchrzak, and J. Dura, Phys. Rev. Lett. 84, 3986 (2000).

[22] M. R. Fitzsimmons, C. Leighton, J. Nogués, A. Hoffmann, Kai Liu, C. F. Majkrzak, J.A. Dura, J. R. Groves, R. W. Springer, P. N. Arendt, V. Leiner, H. Lauter, and I.K. Schuller, Phys. Rev. B 65, 134436 (2002).

[23] S. Widuch, Z. Celinski, K. Balin, R. Schäfer, L. Schultz, D. Skrzypek, and J. McCord, Phys. Rev. B.77, 184433 (2008).

[24] L.Malkinski, T.O'Keevan, R.E.Camley, Z.Celinski, L.Wee, R.L.Stamps, D.Skrzypek, J. Appl. Phys. 93, 6835 (2003)

[25] W.Pang, R.L.Stamps, L.Malkinski, Z.Celinski, D.Skrzypek, J. Appl. Phys. 95, 7309 (2004)

[26] N. C. Koon, Phys. Rev. Lett. 78, 4865 (1997).

[27] T. C. Schulthess, and W. H. Butler, Phys. Rev. Lett. 81, 4516 (1998); J. Appl. Phys. 85, 5510 (1999). 
[28] M. D. Stiles, and R. D. McMichael, Phys Rev. B 59, 3722 (1999).

[29] M. D. Stiles, and R. D. McMichael, Phys. Rev. B 60, 12950 (1999).

[30] R. Camley, and R.L. Stamps, J. Phys.: Condens. Matter 5, 3727 (1993). 